\title{
Isolation, Antibiogram and Molecular Detection of Mannheimia and Pasteurella Associated with Pneumonia in Sheep in Al-Madinah Region, Saudi Arabia
}

\author{
Fayez A Alarawi ${ }^{1}$ and Elhassan MA Saeed ${ }^{2,3^{*}}$
}

\begin{abstract}
${ }^{1}$ Veterinary Diagnostic Laboratory, Branch of the Ministry of Environment, Water and Agriculture, Al Madinah; Saudi Arabia: ${ }^{2}$ Department of Veterinary Medicine, College of Agriculture and Veterinary Medicine, Qassim University, Burraydah, Saudi Arabia: ${ }^{3}$ Department of Microbiology, Faculty of Veterinary Medicine, Khartoum University, Khartoum, Sudan *Corresponding author: esaeed25@gmail.com
\end{abstract}

Article History: 20-243 Received: 10-Dec-2020 Revised: 20-Dec-2020 Accepted: 24-Dec-2020
A BS TRA C T
Pneumonic pasteurellosis is a common and economically important type of ovine pneumonia. No previous study about
the disease in Al Madinah Region, Saudi Arabia. Thus, this study was conducted to determine the association rate of
Mannheimia haemolytica and Pasteurella multocida with pneumonia in sheep and to update data about their
antimicrobial susceptibility pattern. A total of 100 samples (57 nasal swabs and 43 lung tissues) were collected from
diseased and animals suspected to have died of pneumonia. Samples were subjected to bacteriological examination,
biochemical identification of isolates by VITEK2 system, direct molecular identification by real-time PCR (RT-PCR),
and antibiotic sensitivity testing of isolates. The results showed an overall detection rate of $31 \%$ for $M$. haemolytica
(25\%) and P. multocida (6\%). Only $6 \%$ isolates were confirmed by VITEK 2 as $M$. haemolytica, with probability
reached 99\%. While, direct molecular method revealed that $20.2 \%$ samples were positive for $M$. haemolytica and $6.4 \%$
for $P$. multocida specific $16 \mathrm{~S}$ rRNA genes. $M$. haemolytica isolates were found sensitive to oxytetracycline,
nitrofurantoin, trimethoprim/sulfamethoxazole, ciprofloxacin, cefoxitin, ceftazidime, ceftriaxone, imipenem, and
tigecycline, in order. While, they were found completely resistant to cloxacillin, streptomycin, and
amoxicillin/clavulanic acid. In conclusion, the detection rate of $M$. haemolytica emphasized its role as a major cause of
ovine pneumonia. Besides, our results invigorated the role of direct molecular detection and recommend it for laboratory
differential diagnosis. The isolates were resistant to limited antimicrobial agents, nevertheless, the antimicrobial
susceptibility test is important for proper treatment.
Key words: Sheep, Pneumonic pasteurellosis, VITEK2, Antimicrobial, PCR, Al Madinah Region.

\section{INTRODUCTION}

Sheep are the largest and most important livestock resources in Saudi Arabia, with an estimate of 17 million heads (Alghamdi 2016). However, their breeding is threatened by many disease conditions, the most common of which is pneumonia. Though pneumonia is multifactorial, it is mainly caused by bacteria such as Mannheimia haemolytica and Pasteurella multocida, the main causes of pneumonic pasteurellosis in sheep (Mohamed and Abdelsalam 2008; N'jai et al. 2013). P. multocida and $M$. haemolytica are normally found in the upper respiratory tract that can cause pneumonia either alone or in combination with other microbes (Kehrenberg et al. 2005). Their secondary infections are usually predisposed by stressors such as bad weather and shipping, and respiratory pathogens such as Mycoplasma ovipneumoniae, parainfluenza type 3, adenovirus, respiratory syncytial virus, and Bordetella parapertussis (Dassanayake et al. 2010; Marru et al. 2013; El-Yazid et al. 2019). M. haemolytica is commonly isolated from clinical cases, followed by Bibersteinia (Pasteurella) trehalosi and P. multocida (mainly capsular type A) (Jilo et al. 2020).

Pneumonic pasteurellosis is implicated as the cause of considerable economic losses in sheep resulting from mortality and morbidity (Klima et al. 2014). The disease is characterized by an acute febrile course with severe fibrinous or fibrinopurulent bronchopneumonia, fibrinous pleurisy, and septicemia. Infected animals may die within a few days of the onset of clinical signs, but those which survive the acute attack may become chronically infected (Mohamed and Abdelsalam 2008; Abdel-Saeed and Salem, 2019).

Cite This Article as: Alarawi, FA and Saeed EMA, 2021. Isolation, antibiogram and molecular detection of Mannheimia and Pasteurella associated with pneumonia in sheep in Al-Madinah Region, Saudi Arabia. International Journal of Veterinary Science 10(2): 135-140. https://doi.org/10.47278/journal.ijvs/2020.034 
For diagnosis of pneumonic pasteurellosis, isolation of bacteria from nasopharyngeal swabs and lung tissues of suspected animals and biochemical confirmation of isolates is considered the gold standard approach. Recently, molecular methods such as real-time PCR (RT-PCR) have been developed and found to be highly sensitive and specific (Arumugam et al. 2011; Kishimoto et al. 2017). Previous studies on ovine pneumonic pasteurellosis in Saudi Arabia are very scanty (Al-Maary et al. 2017) and little is known about the disease in Al Madinah Region. Hence, this work aimed to elucidate the prevalence of the disease among sheep in Al Madinah Region by isolation and direct molecular detection of $M$. haemolytica and $P$. multocida, and to determine the most effective antimicrobial agents against the isolated strains.

\section{MATERIALS AND METHODS}

\section{Ethical Approval}

Live animals were not used in this study; thus, ethical approval was not needed.

\section{Samples}

A total of 100 samples were collected from different ages of sheep with pneumonia (57 nasal/nasopharyngeal swabs and 43 lung tissues from animals that have died within six hours) in Al-Madinah Region, Saudi Arabia during the period from September 2019 to March 2020. According to clinical examinations, all sheep had signs of anorexia, coughing, dyspnea, lethargy, serous to mucopurulent ocular and nasal discharge, and fever were considered. Samples were obtained from cases attended to veterinary clinics, and from sheep flocks in the area. Samples were collected in sealed sterile labeled containers and transported to Al-Madinah Veterinary Diagnostic Laboratory, Ministry of Environment, Water and Agriculture, for investigation.

\section{Isolation of Pasteurella and Mannheimia Species}

Samples were streaked on $10 \%$ sheep blood agar and MacConkey agar (Oxoid) and incubated aerobically at $37^{\circ} \mathrm{C}$ for $24 \mathrm{~h}$ (Quinn et al. 2011). Suspected colonies were identified by colony characteristics consistent with $M$. haemolytica or P. multocida and cellular morphology in Gram's-stained smears. Gram negative, short to coccobacilli bacteria were further sub-cultured on corresponding agar plates until they were pure (Sharma and Adlakha 1996). Pure cultures of single colony types from both blood and MacConkey agars were transferred to brain heart infusion agar (Oxoid) slants for a series of primary biochemical tests. If the organism can produce a narrow zone of hemolysis on blood agar and grow on MacConkey agar, but unable to produce indole, interpreted as $M$. haemolytica. If the organism is unable to produce hemolysis on blood agar and cannot grow on MacConkey but able to produce indole, interpreted as $P$. multocida (Quinn et al. 2011). Pure colonies were maintained in brain heart infusion agar slants for further identification and confirmation by VITEK 2 and performance of antibiotic sensitivity testing.

\section{Biochemical Confirmation of Isolates}

The obtained isolates were confirmed biochemically using VITEK 2 GN system (BioMérieux) (Avsever 2019).
A suspension from each isolate was prepared from overnight aerobic growth on blood agar plates. An inoculating loop was used to transfer sufficient fresh colonies to a sterile plastic tube containing $3 \mathrm{~mL}$ saline. The suspension was adjusted to a McFarland standard of 0.5 to 0.63 using a Densicheck (BioMérieux). The tube suspensions and GN cards were placed in VITEK 2 cassette and introduced in the VITEK 2 machine for testing. Eventually, the identities of the isolates were obtained with the aid of the accompanying software program.

\section{Antimicrobial Susceptibility Testing}

The obtained isolates were tested for susceptibility to some antimicrobial agents using VITEK 2 AST-N291 system (BioMérieux), which contained 13 antimicrobial agents; namely, nitrofurantoin, trimethoprim/ sulfamethoxazole, ciprofloxacin, cefoxitin, ceftazidime, ceftriaxone, imipenem, tigecycline, ampicillin, meropenem, amikacin, gentamycin, and Amoxicillin/ Clavulanic Acid. For each isolate, about $140 \mu$ inoculum was transferred from previously adjusted suspension of VITEK $2 \mathrm{GN}$ system to a new plastic tube containing $3 \mathrm{~mL}$ sterile saline. The tube suspensions and AST-N291 cards were placed in VITEK 2 cassette and introduced in the VITEK 2 machine for testing. For more antimicrobial agents, streptomycin (S10), cloxacillin (OB5), penicillin-G (P10), oxytetracycline (OT30), and erythromycin (E15) were also tested using the standard disk diffusion method. The zone of inhibition was interpreted based on the Performance Standards for Antimicrobial Susceptibility (Barry 2007).

\section{Direct Molecular Detection of Pasteurella and Mannheimia spp.}

DNA was extracted directly from collected samples using PureLink ${ }^{\circledR}$ Genomic DNA Kit (Invitrogen, Life Technologies, USA) following the manufacturer's instructions. Real-time PCR methods for the detection of species-specific genes for $P$. multocida and $M$. haemolytica were performed using the primers and probes of BactoReal ${ }^{\circledR}$ Kit (ingenetix, GmbH, Arsenalstraße 11, 1030 Vienna, Austria). BactoReal ${ }^{\circledR}$ Kit M. haemolytica and BactoReal ${ }^{\circledR}$ Kit $P$. multocida were used for detection of 16S rDNA gene of $M$. haemolytica and $P$. multocida, respectively. A probe-specific amplification-curve at 530nm (FAM channel) indicated the amplification of $M$. haemolytica and P. multocida specific DNA (BactoReal ${ }^{\circ}$ Kit-P. multocida and M. haemolytica Manuals).

One microlitre from primer and probe Assay Mix was put in a $20 \mu 1$ reaction volume that contained $10 \mu 1$ of DNA Reaction Mix, $4 \mu 1$ PCR grade water, and $5 \mu 1$ extracted DNA from samples. For negative and positive controls, template DNA was replaced by PCR grade water and positive Control-DNA in the same purchased kit respectively. PCR was conducted on a LightCycler $^{\circledR}$ Carousel-Based System apparatus (Roche Applied Science, Mannheim, Germany) using cycling conditions that are displayed in Table 1 . The hydrolysis probe format was used for real-time PCR detection. The data was analyzed using the second derivative maximum method and viewed at detection format channel 530nm (FAM channel). 


\section{RESULTS}

\section{The Isolation Rate of Pasteurella Species}

The isolation method revealed $6 \% \mathrm{M}$. haemolytica isolates, which were identified by colony morphology and microscopic characteristics; and confirmed by VITEK 2 compact system. Four isolates from nasal swabs (7\%), and two from lung tissues $(4.7 \%)$.

\section{Morphological Characteristics of M. Haemolytica Isolates}

The suspected colonies were identified by characteristic colony morphology and Gram staining. The Gram-stained smears from the purified colonies showed Gram-negative coccobacilli, arranged singly or in pairs (Fig. 1A). The recovered colonies' growth on blood agar appeared as small, round, smooth, convex, and opaque, with beta hemolysis (Fig. 1B). On MacConkey agar, their growth was red in color due to lactose fermentation (Fig. 1C).

\section{Biochemical Characterization of the Isolates}

All of the six recovered isolates were confirmed biochemically by VITEK 2 compact system as $M$. haemolytica using GN card; with excellent identification (probability reached $99 \%$ for four of them, while the other two $92 \%$ and $93 \%$ ).

Antibiotic Sensitivity Testing of $M$. haemolytica Isolates

The six obtained isolates were tested for their susceptibility to antimicrobial agents by two methods: Kirby-Bauer disk diffusion method and Vitek 2 Compact AST cards. The isolates of $M$. haemolytica were found highly sensitive to oxytetracycline, nitrofurantoin, trimethoprim/ sulfamethoxazole, ciprofloxacin, cefoxitin, ceftazidime, ceftriaxone, imipenem, tigecycline; intermediately sensitivity to: erythromycin, penicillin-G, ampicillin, meropenem, amikacin, gentamycin; and resistant to cloxacillin, streptomycin, and amoxicillin/clavulanic acid.

\section{Direct Molecular Detection of Pasteurella multocida and M. haemolytica}

The DNA was isolated directly from all clinical samples, except the six ones from which isolates were obtained, i.e. from 94 samples, which were screened by RT-PCR for the presence of $M$. haemolytica and $P$. multocida specific $16 \mathrm{~S}$ rRNA genes. The obtained results revealed that 19 samples $(20.2 \%)$ were positive for $M$. haemolytica and six samples $(6.4 \%)$ were positive for $P$. multocida (Fig. 2; Fig. 3).

The total detection rate (isolation and molecular) of $M$. haemolytica was $25 \%$ and that of $P$. multocida was $6 \%$ (Table 2). No sample showed the presence of both $M$. haemolytica and P. multocida. Thus, $31 \%$ of animals investigated have pneumonic pasteurellosis.

\section{DISCUSSION}

Sheep are the main and most important source of meat production in Saudi Arabia. However, they are suffering from various disease conditions, most notably pneumonia caused by Pasteurella species (pneuomonic pasteurellosis). Pneumonia is known as a multifactorial disease (N'jai et al. 2013), but despite that differential laboratory diagnosis is

Table 1: Cycling conditions of LightCycler ${ }^{\circledR}$ RT-PCR for detection of M. haemolytica and P. multocida using BactoReal ${ }^{\circledR}$ Kit Program BactoReal ${ }^{\circledR}$ Kit $\quad$ Program1 Program2 Program3

\begin{tabular}{lcccc} 
Program & & & & \\
\hline Analysis Mode & None & None & \multicolumn{2}{c}{ Quantification Mode } \\
Cycles & 1 & 1 & \multicolumn{2}{c}{45} \\
Target & 50 & 95 & 95 & 60 \\
Hold [hh:mm:ss] & $0: 02: 00$ & $0: 00: 20$ & $00: 00: 05$ & $0: 01: 00$ \\
Ramp rate $\left[{ }^{\circ} \mathrm{C} / \mathrm{s}\right] 96$ & 4.4 & 4.4 & 4.4 & 2.2 \\
Acquisition Mode & None & None & None & Single \\
\hline
\end{tabular}

Table 2: Comparison between detection rate of $M$. haemolytica and P. multocida according to diagnostic method and type of samples

\begin{tabular}{llccc}
\hline \multirow{2}{*}{$\begin{array}{l}\text { Bacterial } \\
\text { species }\end{array}$} & Sample type & \multicolumn{2}{c}{ Number of positive samples } & Total \\
\cline { 3 - 4 } & & Culture method & PCR & $(\%)$ \\
\hline$M$. & Nasal swabs & 4 & 14 & 18 \\
haemolytica & Lung tissues & 2 & 5 & 7 \\
P. multocida & Nasal swabs & 0 & 0 & 0 \\
& Lung tissues & 0 & 6 & 6 \\
Total & & 6 & 25 & 31 \\
\hline
\end{tabular}

A

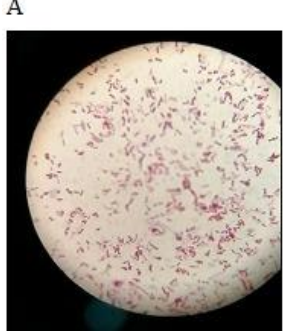

B
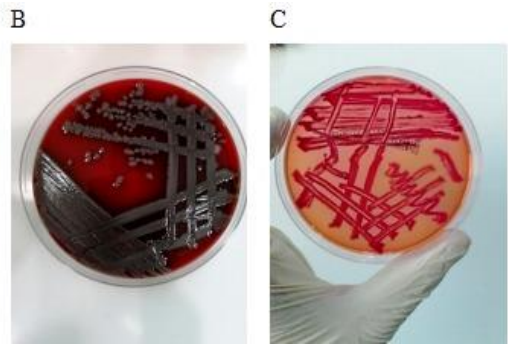

Fig. 1: (A) Microscopical appearance of Gram-stained $M$ haemolytica coccobacilli, arranged singly or in pairs; (B) Colonies of $M$. haemolytica on blood agar appeared as small, round, smooth, convex and opaque; (C) Growth of $M$. haemolytica on MacConkey agar showing lactose-fermentation property (red color).

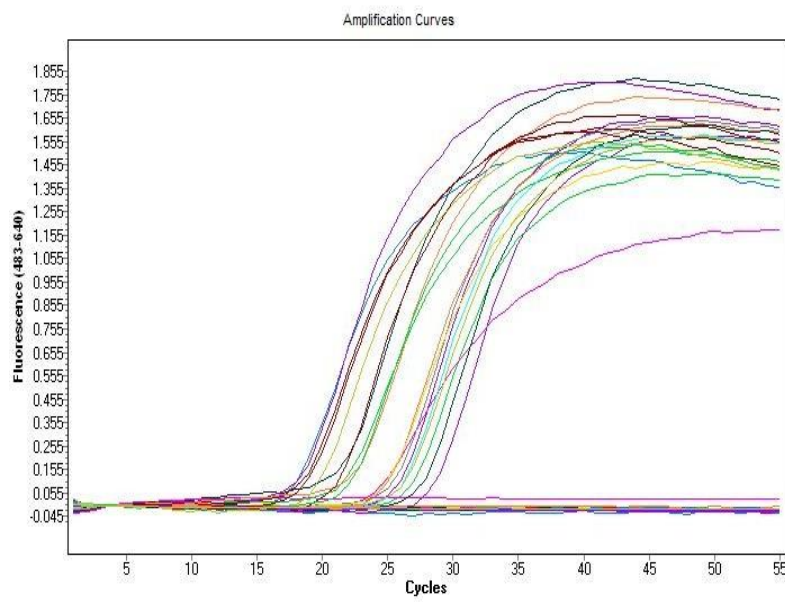

Fig. 2: Real-time PCR amplification plots for detection of $16 \mathrm{~S}$ rDNA gene specific for $M$. haemolytica obtained from nasal and lung tissues from suspected cases of pneumonic pasteurellosis in sheep. Negative and positive controls are represented by PCR water and DNA of BactoReal ${ }^{\circledR}$ Positive Control for $M$. haemolytica, respectively. Nineteen samples were positive for $M$. haemolytica.s

rarely practiced and treatment is mainly prescribed against Pasteurella spp.; at least in the area of our study. No previous study was found in Al Madinah Region about the diagnosis of the disease and its treatment. Thus, the present 


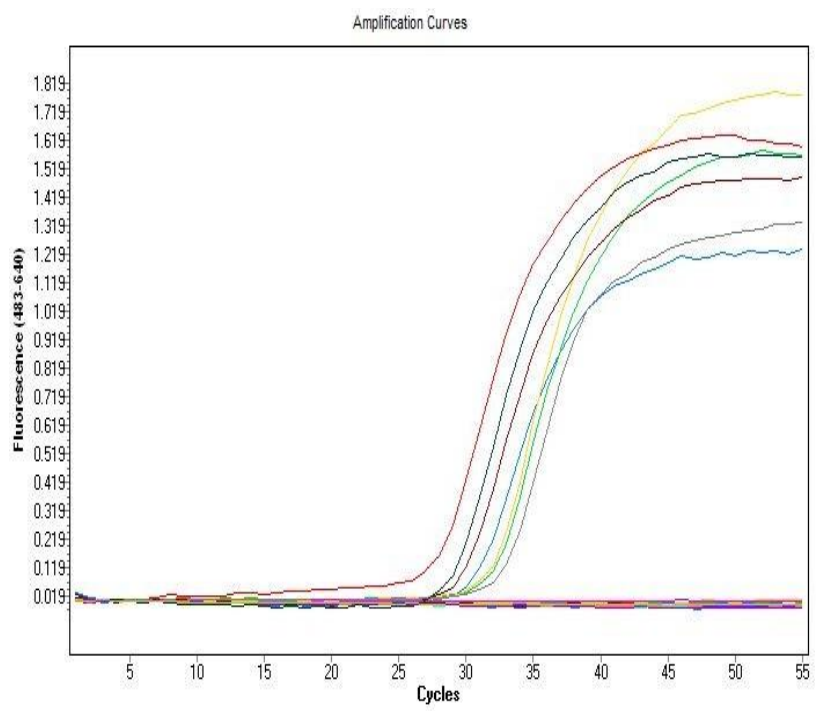

Fig. 3: Real-time PCR amplification plots for detection of $16 \mathrm{~S}$ rDNA gene specific for $P$. multocida obtained from lung tissues from suspected cases of pneumonic pasteurellosis in sheep. Negative and positive controls are represented by PCR water and DNA of BactoReal® Positive Control for $P$. multocida, respectively. Six samples were positive for $P$. multocida

study was undertaken to determine the association rate of $P$. multocida and $M$. haemolytica with suspected pneuomonic pasteurellosis in sheep; and to assess their sensitivity to the commonly used antimicrobial agents.

In the present study, the overall detection rate of $P$. multocida and $M$. haemolytica was found $31 \%$ of investigated samples. M. haemolytica was isolated and confirmed by VITEK 2 system from six samples (6\%); and its specific 16S rRNA gene was detected in other 19 samples by direct RT-PCR, with an overall detection rate of $25 \%$. While, P. multocida was not isolated, but its specific $16 \mathrm{~S}$ rRNA gene was detected by direct RT-PCR in six $(6 \%)$ samples. This overall detection rate $(31 \%)$ is following some previous studies (Tilaye 2010; Alemneh and Tewodros 2016), which have found prevalence rates of 32.6 and $28.4 \%$, respectively. However, the majority of previous studies have reported higher prevalence rates (Gilmour 1974; Aschalew 1998; Singh et al. 2018). This variation in detection rates might be due to the ecology of the study areas, seasonal variation, and the presence of predisposing factors (Abera et al. 2014). In the current study, $M$. haemolytica was found more prevalent than $P$. multocida, a finding most commonly found previously (Mohammed 1999; Marru et al. 2013; Alemneh and Tewodros 2016; Sahay et al. 2020). In contrast, BlancoViera et al. (1995) found P. multocida more prevalent in sheep and cattle than $M$. haemolytica. The high prevalence of $M$. haemolytica may indicate that it is the major causative agent of ovine pneumonia in the area of our study. M. haemolytica, which is a normal flora of the upper respiratory tract, may play a secondary role after a primary initiating agent that can suppress the host defense mechanism, and enhance the multiplication of $M$. haemolytica or other Pasteurella species leading to bronchopneumonia (Aiello and Mays 1998). The combination of stressors and primary infections are thought to break down the mucosal barrier integrity of the lower respiratory tract, allowing $M$. haemolytica to colonize, proliferate, and induce significant tissue damage (Ackermann 1996).

In the current study, more than 40 suspected Pasteurella-like colonies and cells were isolated. Nevertheless, only six $(6 \%)$ were confirmed as $M$. haemolytica by VITEK 2 and no isolate of $P$. multocida was obtained. The current isolation rate is lower than those reported by Kaoud et al. (2010), Marru et al. (2013) and Alemneh and Tewodros (2016), who stated 14.1, 25 and $32.6 \%$, respectively. However, a similar isolation rate is found reported by Sahay et al. (2020), who reported 7.4\% for $M$. haemolytica. These variations are likely to be caused by several factors including the variation of microbial carriage in the respiratory tract in different niches, different isolation techniques, misidentification and contamination by other swarming bacteria. Also, it has been found that the prevalence of $M$. haemolytica in temperate climates varies seasonally with a higher prevalence in spring and early summer (Adlam and Rutter 1989).

Molecular screening of all collected samples (except ones from which isolates were obtained) was performed using RT-PCR with specific primers and probes targeting $16 \mathrm{~S}$ rRNA of $M$. haemolytica and $P$. multocida. DNA templates were obtained directly from samples. Nineteen samples $(20.2 \%)$ were positive for M. haemolytica and other six samples were positive for $P$. multocida $(6.4 \%)$ (Fig. 2 and 3). These samples $(19+6=25)$ were all negative by isolation method, indicating that molecular detection is more sensitive and specific, and should always be preferred to isolation and biochemical identification method, which, in addition, requires strict sterile handling and takes much more time. Similarly, Sahay et al. (2020) have reported a higher detection rate for $M$. haemolytica $(66.6 \%)$ and $P$. multocida $(19.1 \%)$ by direct RT-PCR compared to $7.2 \%$ and $7.4 \%$ isolation rate, respectively. The $16 \mathrm{~S}$ rRNA gene sequencing has been used as the gold standard for precise molecular identification and phylogenetic relationship studies of $P$. multocida and other species (Davies et al. 2003). For Mannheimia, the 16S rRNA is still considered the gold standard, however, sodA gene sequencing showed a markedly higher divergence and ability to differentiate related species than the corresponding $16 \mathrm{~S}$ rRNA gene (Guenther et al. 2008).

The detection rate of bacteria according to sample type indicated the presence of $M$. haemolytica in nasal swabs more than in lung tissues. This finding may indicate that its association is partially causal. On the contrary, $P$. multocida was detected in lung tissues only, a finding may indicate that its association is mostly causal.

Changes in susceptibility to antimicrobial agents for both $P$. multocida and $M$. haemolytica isolates have been reported (Catry et al. 2006). Hence, it is important to monitor the antimicrobial susceptibility of Pasteurella species to determine resistance development. Marru et al. (2013) stated that despite the diversity in the site of origins, $P$. multocida and $M$. haemolytica isolates exhibited uniformity in sensitivity to a majority of the antibacterial agents. In the present study, the isolates of $M$. haemolytica were tested for their susceptibility to 18 antimicrobial agents. They were found resistant to three of them (cloxacillin, streptomycin, and amoxicillin/clavulanic acid), intermediately sensitive to six (erythromycin, penicillin-G, ampicillin, meropenem, amikacin, and 
gentamycin), and highly sensitive to the rest nine. The zone of inhibition was interpreted based on the Performance Standards for Antimicrobial Susceptibility (Barry 2007). Our findings indicated that antibiotic therapy is still a good control measure in light of the presence of several highly effective antibiotics. However, due to the presence of some ineffective drugs, the use of research outcomes on susceptibility to antibiotics and/or conduction of antibiotic sensitivity tests remain necessary. The present study results are partially consistent with previous studies; e.g., Marru et al. (2013) have found sulfamethoxazole and tetracycline as most effective, ampicillin as intermediately effective, and streptomycin as an ineffective drug against $M$. haemolytica; a finding in agreement with ours. Unlikely, they found resistance to gentamycin and penicillin-G, while we found intermediate sensitivity to them. In contrast, Seker et al. (2009) found gentamycin as the most effective drug against $M$. haemolytica. Similarly, Post et al. (1991) reported that $90 \%$ of $M$. haemolytica isolates recovered from cattle with bovine respiratory disease complex were markedly susceptible to gentamycin. Also, a result similar to ours was reported for penicillin, gentamicin, and streptomycin, and contradictory to ours for oxytetracycline by Sahay et al. (2020). In the current study, $M$. haemolytica was found resistant to amoxicillin/ clavulanic acid, while it was found sensitive to it in a previous study (Sebbar et al. 2019). Contradiction in findings between various studies might be due to difference in the strain of the isolate that may cause pasteurellosis in different species of animals, or due to the existence of host factors that may affect the action of the drug in sheep (Marru et al. 2013). Besides, the level of misuse of drugs is expected to vary from a region to another, which may have a role in variation in the development of resistance to antibiotics (Mehmood et al. 2020). These differences fortify the recommendation of Önat et al. (2010) which stated that "antibiotic susceptibility profiles of $P$. multocida and $M$. haemolytica help veterinarians to choose appropriate antibiotic against bovine respiratory disease; however, antibiotic susceptibility studies should be renewed periodically".

\section{Conclusion}

In conclusion, $M$. haemolytica was found as a major cause of ovine pneumonia in the area where this study was undertaken, whereas $P$. multocida is uncommon. Their presence in only $31 \%$ of suspected pneumonic pasteurellosis cases emphasizes the importance of proper clinical and laboratory differential diagnosis. Direct molecular detection was found efficient; therefore, we recommend using it for a routine examination. The antibiogram results disclosed the current antibiotic susceptibility pattern of these bacteria, therefore, we recommend for local animal health authorities to consider our results for treatment of suspected pneumonic pasteurellosis cases.

\section{Acknowledgment}

The authors would like to thank the Director-General of the Branch of Ministry of Environment, Water and Agriculture, Al Madinah Region, the Director of Animal Resources Directorate, and the Director of Al Madinah Diagnostic Veterinary Laboratory for their permission to do part of this work in the laboratory.

\section{Authors' Contribution}

EMA Saeed provided the original idea, and both authors designed the study. FAA collected the samples and performed the laboratory work. The manuscript was drafted by EMA Saeed and revised and approved by the two authors.

\section{REFERENCES}

Abera D, Sisay $\mathrm{T}$ and Birhanu, T, 2014. Isolation and identification of Mannhemia and Pasturella species from pneumonic and apparently healthy cattle and their antibiogram susceptibility pattern in Bedelle District, Western Ethiopia. African Journal of Bacteriology Research 6: 32-41. https://doi.org/10.5897/JBR2014.0143

Abdel-Saeed H and Salem NY, 2019. Evaluation of total antioxidant capacity, malondialdehyde, catalase, proteins, zinc, copper and $\operatorname{IgE}$ response in ovine verminous pneumonia. International Journal of Veterinary Science 8: 255-258.

Ackermann MR, Kehrli Jr ME and Brogden KA, 1996. Passage of CD 18- and CD 18+ bovine neutrophils into pulmonary alveoli during acute Pasteurella haemolytica pneumonia. Veterinary Pathology 33: 639-646. https://doi.org/10.1177/ 030098589603300602

Adlam C and Rutter J, 1989. Pasteurella and pasteurellosis. Academic Press, London, UK.

Aiello SE and Mays A, 1998. The Merck Veterinary Manual. Merck \& Co. Inc., Whitehouse Station, NJ, pp: 2032-2033.

Alemneh T and Tewodros A, 2016. Sheep and goats pasteurellosis: Isolation, identification, biochemical characterization and prevalence determination in Fogera Woreda, Ethiopia. Journal of Cell and Animal Biology 10: 22-29. https://doi.org/10.5897/JCAB2016.0449

Alghamdi KA, 2016. Designing geodatabases for the general authority for statistics of the Kingdom of Saudi Arabia http://hdl.handle.net/1969.6/684

Al-Maary KS, Dawoud TM, Mubarak AS, Hessain AM, Galal HM, Kabli SA and Mohamed MI, 2017. Molecular characterization of the capsular antigens of Pasteurella multocida isolates using multiplex PCR. Saudi Journal of Biological Sciences 24: 367-370. https://doi.org/10.1016/ j.sjbs.2016.06.006

Arumugam N, Ajam N, Blackall P, Asiah N, Ramlan M, Maria J, Yuslan S and Thong KL, 2011. Capsular serotyping of Pasteurella multocida from various animal hosts-a comparison of phenotypic and genotypic methods. Tropical Biomedicine 28: 55-63.

Aschalew Z, 1998. A study on pneumonic pasteurellosis in North Shewa. Debre-Zeit: DVM thesis, FVM, Addis Ababa University, Ethiopia, pp: 59.

Avsever ML, 2019. Bacterial infections and antibiogram results of farm, pet and other some animals in the Aegean Region. Van Veterinary Journal 30: 95-101.

Barry AL, 2007. Chapter 1. An Overview of the Clinical and Laboratory Standards Institute (CLSI) and its impact on antimicrobial susceptibility tests. In: Schwalbe R, SteelMoore L and Goodwin AC (ed), Antimicrobial Susceptibility Testing Protocols, $1^{\text {st }}$ Ed. Boca Raton, FL: CRC Press, pp: 1-6.

Blanco-Viera F, Trigo F, Jaramillo-Meza L and Aguilar-Romero F, 1995. Serotypes of Pasteurella multocida and Pasteurella haemolytica isolated from pneumonic lesions in cattle and sheep from México. Revista Latinoamericana de Microbiologia 37: 121-126.

Catry B, Decostere A, Schwarz S, Kehrenberg C, de Kruif A and Haesebrouck F, 2006. Detection of tetracycline-resistant and susceptible Pasteurellaceae in the nasopharynx of loose group-housed calves. Veterinary Research Communications 30: 707-715. https://doi.org/10.1007/s11259-006-3347-8 
Dassanayake RP, Shanthalingam S, Herndon CN, Subramaniam $\mathrm{R}$, Lawrence PK, Bavananthasivam J, Cassirer EF, Haldorson GJ, Foreyt WJ, Rurangirwa FR, Knowles DP, Besser TE and Srikumaran S, 2010. Mycoplasma ovipneumoniae can predispose bighorn sheep to fatal Mannheimia haemolytica pneumonia. Veterinary Microbiology 145: 354-359. https://doi.org/10.1016/j. vetmic.2010.04.011

Davies R, Caffrey B and Watson P, 2003. Comparative analyses of Pasteurella multocida strains associated with the ovine respiratory and vaginal tracts. Veterinary Record 152: 7-10. http://doi.org/10.1136/vr.152.1.7

El-Yazid HA, Soliman R, Wasif IM, El Kariem Selim SA, Balata M, Mahmood Z and Seida AA, 2019. Protective efficacy of the inactivated adjuvant vaccines against Mycoplasma agalactiae infection in goats. International Journal of Veterinary Science 8: 14-19.

Gilmour N, 1974. The recovery of Pasteurella haemolytica from the tonsils of adult sheep. Research in Veterinary Science 17: 413-414. https://doi.org/10.1016/S0034-5288(18)33671-3

Guenther S, Schierack P, Grobbel M, Lübke-Becker A, Wieler LH and Ewers C, 2008. Real-time PCR assay for the detection of species of the genus Mannheimia. Journal of Microbiological Methods 75: 75-80. https://doi.org/10.1016/ j.mimet.2008.05.008

Jilo K, Belachew T, Birhanu W, Habte D, Yadeta W and Giro A, 2020. Pasteurellosis status in Ethiopia: A comprehensive review. Journal of Tropical Diseases and Public Health 8: 1-5.

Kaoud H, El-Dahshan A, Zaki M and Nasr SA, 2010. Occurrence of Mannheimia haemolytica and Pasteurella trehalosi among ruminants in Egypt. New York Science Journal 3: 135-141.

Kehrenberg C, Catry B, Haesebrouck F, De Kruif A and Schwarz $\mathrm{S}, 2005$. tet (L)-mediated tetracycline resistance in bovine Mannheimia and Pasteurella isolates. Journal of Antimicrobial Chemotherapy 56: 403-406. https://doi.org/ 10.1093/jac/dki210

Kishimoto M, Tsuchiaka S, Rahpaya SS, Hasebe A, Otsu K and Sugimura S, 2017. Development of a one-run real-time PCR detection system for pathogens associated with bovine respiratory disease complex." Journal of Veterinary Medical Science 79: 517-723. https://doi.org/10.1292/jvms.16-0489

Klima CL, Alexander TW, Hendrick S and McAllister TA, 2014. Characterization of Mannheimia haemolytica isolated from feedlot cattle that were healthy or treated for bovine respiratory disease. Canadian Journal of Veterinary Research 78: 38-45.

Marru HD, Anijajo TT and Hassen AA, 2013. A study on ovine pneumonic pasteurellosis: isolation and identification of pasteurellae and their antibiogram susceptibility pattern in Haramaya District, Eastern Hararghe, Ethiopia. BMC Veterinary Research 9: 1-8. https://doi.org/10.1186/17466148-9-239

Mehmood K, Bilal RM and Zhang H, 2020. Study on the genotypic and phenotypic resistance of tetracycline antibiotic in Escherichia coli strains isolated from free ranging chickens of Anhui Province, China. Agrobiological Records 2: 63-68. https://doi.org/10.47278/ journal.abr/2020.014
Mohamed RA and Abdelsalam EB, 2008. A review on pneumonic pasteurellosis (respiratory mannheimiosis) with emphasis on pathogenesis, virulence mechanism and predisposing factors. Bulgarian Journal of Veterinary Medicine 11: 139160.

Mohammed R, 1999. Bacteriological and histological examination of pneumonic lungs of small ruminants slaughtered at Hashim Nure export abattoir. DVM thesis, AAU, FVM. Debre Zeit, Ethiopia, pp: 1-21.

N'jai AU, Rivera J, Atapattu DN, Owusu-Ofori K and Czuprynski CJ, 2013. Gene expression profiling of bovine bronchial epithelial cells exposed in vitro to bovine herpesvirus 1 and Mannheimia haemolytica. Veterinary Immunology and Immunopathology 155: 182-189. https://doi.org/10.1016/ j.vetimm.2013.06.012

Önat K, Kahya S and Çarli KT, 2010. Frequency and antibiotic susceptibility of Pasteurella multocida and Mannheimia haemolytica isolates from nasal cavities of cattle. Turkish Journal of Veterinary and Animal Sciences 34: 91-94. https://doi.org/10.3906/vet-0901-1

Post KW, Cole NA and Raleigh RH, 1991. In vitro antimicrobial susceptibility of Pasteurella haemolytica and Pasteurella multocida recovered from cattle with bovine respiratory disease complex. Journal of Veterinary Diagnostic Investigation 3: 124-126. https://doi.org/10.1177/1040638 $\underline{79100300203}$

Quinn PJ, Markey BK, Leonard FC, Hartigan P, Fanning S and Fitzpatrick E, 2011. Veterinary Microbiology and Microbial Disease. $2^{\text {nd }}$ Ed. John Wiley \& Sons.

Sahay S, Natesan K, Prajapati A, Kalleshmurthy T, Shome BR, Rahman H and Shome R, 2020. Prevalence and antibiotic susceptibility of Mannheimia haemolytica and Pasteurella multocida isolated from ovine respiratory infection: A study from Karnataka, Southern India. Veterinary World 13: 1947 1954. http://doi.org/10.14202/vetworld.2020.1947-1954

Sebbar G, Zro K, Id Sidi Yahia K, Elouennass M, Filali-Maltouf A and Belkadi B, 2019. Antimicrobial susceptibilty screening test of $M$. haemolytica and $P$. multocida (serogroup A) moroccan strains isolated from ruminants. Bulgarian Journal of Veterinary Medicine. https://doi.org/ 10.15547/bjvm.2019-0109

Seker E, Kuyucuoglu Y and Konak S, 2009. Bacterial examinations in the nasal cavity of apparently healthy and unhealthy Holstein cattle. Journal of Animal and Veterinary Advances 8: 2355-2359.

Sharma S and Adlakha S, 1996. Textbook of Veterinary Microbiology. $1^{\text {st }}$ ed. Vikas Publishing House Pvt Ltd., New Delhi, India.

Singh F, Sonawane GG and Meena RK, 2018. Molecular detection of virulent Mannheimia haemolytica and Pasteurella multocida in lung tissues of pneumonic sheep from semiarid tropics, Rajasthan, India. Turkish Journal of Veterinary and Animal Sciences 42: 556-561. https://doi.org/ 10.3906/vet-1802-73

Tilaye D, 2010. Pathological, bacteriological and serological study on pneumonic sheep and goats with special emphasis on Mannheimia, Bibersteinia, Pasteurella and Mycoplasma species. MSc Thesis, AAU, FVM, Debre Zeit, Ethiopia, pp. $1-97$. 